\title{
Live Birth in Sex-Reversed XY Mice Lacking the Nuclear Receptor Dax1
}

\author{
Isabel Fernandes-Freitas ${ }^{1,4}$, Alexandra Milona ${ }^{2,3,4}$, Kevin G. Murphy ${ }^{1}$, Waljit S. Dhillo ${ }^{1 *} \&$ \\ Bryn M. Owen ${ }^{1^{*}}$
}

The nuclear hormone receptor Dax1 functions during development as a testes-determining gene. However, the phenotype of male mice lacking Dax1 is strain-dependent due to the background-specific abundance of male-determining Sry gene-transcripts. We hypothesised that inter-individual variation in SrymRNA-abundance would result in a spectrum of phenotypes even within-strain. We found that while all XY C57BL/6J mice lacking Dax1 presented as phenotypic females, there was a marked interindividual variability in measures of fertility. Indeed, we report rare occasions where sex-reversed mice had measures of fertility comparable to those in control females. On two occasions, these sex-reversed $\mathrm{XY}$ mice were able to give birth to live offspring following mating to stud-males. As such, this work documents within-strain variability in phenotypes of XY mice lacking Dax1, and reports for the first time a complete sex-reversal capable of achieving live birth in these mice.

Dax1 is an orphan member of the nuclear-receptor class of transcription factors ${ }^{1}$. Its gene, $N r 0 b 1$, is found on the X-Chromosome and encodes a 470 amino acid protein consisting of an N-terminal DNA-binding domain, and a C-terminal region containing a putative ligand-binding domain. Classically, Dax1 represses the transcriptional-activation function of nuclear receptors through protein-protein interactions ${ }^{2}$. However, it has also been reported to activate transcription, and have other functions through its ability to directly bind DNA ${ }^{3}$. In adults, Dax1 is expressed throughout the hypothalamic-pituitary-gonadal (HPG) axis ${ }^{4}$. It interacts with several steroidogenic receptors including those for estrogen and testosterone ${ }^{5}$. However, the role of Dax1 in the adult HPG axis is largely unknown.

During development, Dax1 is a testes-determining gene ${ }^{3,6,7}$. While XX Dax1-KO mice are overtly fertile, XY mice lacking Dax1 display a spectrum of sex-reversal phenotypes apparently depending on the animal's background-strain. For example, XY 129 Sv/J mice lacking exon-2 of Dax1 have abnormal testicular development resulting in smaller than normal adult testes ${ }^{8}$. By contrast, XY mice lacking Dax1 in a mixed genetic background, and containing the 'weak' Sry allele from the Mus domesticus poschiavinus Y-Chromosome, display complete sex-reversal ${ }^{6,7}$. Furthermore, ovary development in B6 mice lacking Dax 1 can be prevented by the addition of multiple copies of the male-determining Sry allele from the 129-strain ${ }^{7}$. As such, it has been suggested that Dax1-deficiency can be bypassed by overexpression of Sry gene-transcripts. Importantly, developmental Sry-expression is also variable between individuals of the same strain ${ }^{7}$. It is therefore possible that a range of sex-reversal phenotypes may occur even within-strain in XY Dax1-KO mice. However, this has not previously been investigated.

We surveyed reproductive function in chromosomal-XY C57BL/6J mice lacking exon 2 of the Dax 1 gene, henceforth referred to as Dax $1-/ Y$. At weaning, all of these animals were phenotypically female. However, they displayed a spectrum of reproductive phenotypes and molecular measures of fertility. Many Dax $1-/ Y$ mice were completely infertile. However, a minority of sex-reversed chromosomal-XY Dax1-/Y mice had measures of fertility comparable to those in control female mice. Indeed, two out of a total of 18 Dax1-/Y mice became pregnant and gave birth to live offspring after mating with stud-males. As such, this work demonstrates intra-strain variability in sex-reversal phenotypes of chromosomal-XY mice lacking Dax1. It also reports for the first time a live birth from a sex-reversed chromosomal-male mouse lacking this orphan nuclear receptor.

\footnotetext{
${ }^{1}$ Section of Endocrinology \& Investigative Medicine, Division of Diabetes, Endocrinology, and Metabolism, Department of Metabolism, Digestion, and Reproduction, Imperial College London, London, United Kingdom. ${ }^{2} \mathrm{MRC}$ London Institute of Medical Sciences (LMS), London, United Kingdom. ${ }^{3}$ Institute of Clinical Sciences (ICS), Faculty of Medicine, Imperial College London, London, United Kingdom. ${ }^{4}$ These authors contributed equally: Isabel FernandesFreitas and Alexandra Milona. *email:w.dhillo@imperial.ac.uk; bryn.owen05@imperial.ac.uk
} 


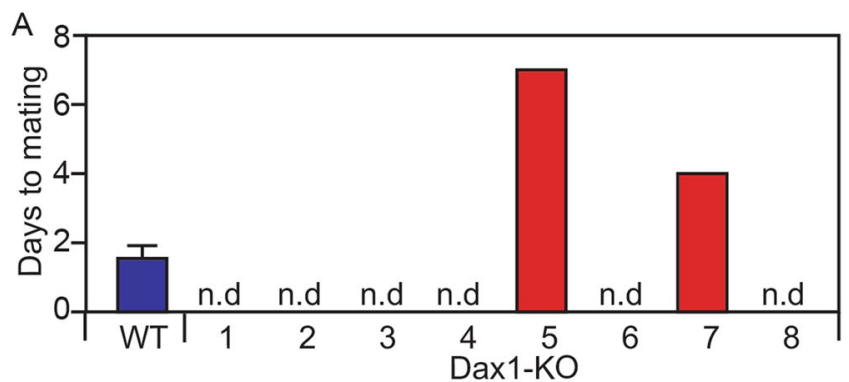

B

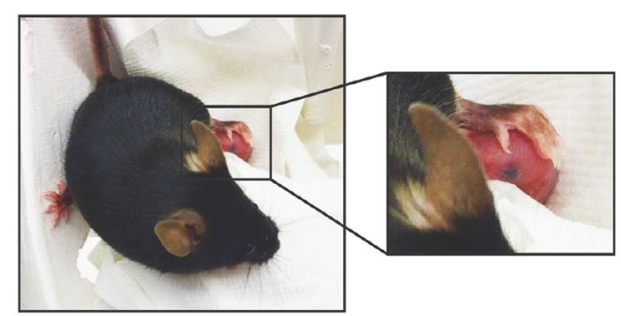

Figure 1. Live-birth in a Dax $1-/ Y$ mouse. (A) Mating success of four WT $($ Dax $1+/+)$ females presented as average $+/-$ SEM and eight Dax $1-/ Y$ mice with proven stud-males. The $Y$ axis shows the number of days that a test-mouse was mated with a stud-male before a vaginal plug was detected. (B) Photograph of a Dax1-/Y mouse with single live-offspring on post-natal day 1 .

\section{Results}

We generated C57BL/6J mice lacking exon 2 of the Dax1 (NrOb1) gene, as described ${ }^{7}$. In accordance with previous findings, all of these Dax1-/Y mice presented as phenotypic females at weaning.

In a preliminary experiment to test fertility, we paired 10 Dax $1-/ Y$ mice with proven $\operatorname{Dax} 1+/ Y$ stud-males. Approximately two months after pairing, one Dax1-/Y mouse gave birth to a single offspring which was found dead in the home-cage (data not shown). To confirm this finding, we set up a second fertility experiment involving eight Dax $1-/ Y$ mice and four control $(\operatorname{Dax} 1+/+)$ females. All of the control female mice mated with the stud-males (Fig. 1A). By contrast, only two out of eight Dax1-/Y mice mated with the stud-males during the 10-day test period (Fig. 1A). One of these animals became pregnant and gave birth to an apparently healthy single offspring (Fig. 1B). For an undetermined reason, the pup did not survive beyond postnatal day two. Therefore, a minority of sex-reversed Daxl-/Y mice can give birth to live offspring.

The results of our mating-experiments were suggestive of intra-strain variability in the sex-reversal phenotype of Dax 1-/Y mice. In order to investigate the spectrum of reproductive phenotypes in Dax $1-/ Y$ mice, we conducted experiments in an additional cohort of four control Dax $1+/+$ female mice and eight sex-reversed Dax 1-/Y mice. We first assessed the timing of puberty by determining vaginal opening ${ }^{9}$. Control females underwent vaginal opening at approximately 21 days of age (Fig. 2A). By contrast, four out of eight Dax $1-/ Y$ mice did not undergo vaginal opening at all during the 40-day test-period (Fig. 2A). Three Dax $1-/ Y$ mice underwent a delayed vaginal opening (between day-26 and day-35), and one Dax $1-/ Y$ mouse underwent normal vaginal opening on day-20 (Fig. 2A).

We next assessed the estrous cyclicity by vaginal cytology. As expected, control female mice underwent a 4-6 day cycle and entered the post-ovulatory estrous-phase twice during the 11-day testing period (Fig. 2B). By contrast, over half (five out of eight) of the Dax1-/Y mice did not enter the ovulatory phase of the cycle at all during the testing-period (Fig. 2C). Two Dax1-/Y mice did enter the estrous-phase but did not cycle normally. One Dax $1-/ Y$ mouse cycled normally and entered the estrous-phase twice during the test-period. There was a clear connection between the Dax1-/Y individuals that underwent vaginal opening (Fig. 2A) and those that had evidence of estrous cyclicity (Fig. 2C). In addition, the gross morphology of the uterus and ovaries reflected the vaginal cytology data. Those animals displaying evidence of estrous cyclicity had larger ovaries and uteri than those that did not (Fig. 2C). Together, these data show clear intra-strain variability in the reproductive phenotype of C5BL/6J Dax1-/Y mice. A minority of sex-reversed Dax1-/Y mice are comparable to control females with regard to their time of puberty onset and estrous cyclicity.

In order to gain additional insight in to our observations on sex-reversal phenotypes, we conducted post-mortem analysis of plasma hormone levels, ovaries, and hypothalamic neuropeptide gene-expression. Plasma estrogen (E2), luteinizing hormone (LH), and follicle-stimulating hormone (FSH) were all significantly lower $(\mathrm{p}<0.05)$ in Dax $1-/ Y$ mice than in control females. Indeed, these hormones were undetectable in at least half of the Dax1-/Y mice (Fig. 3A,B). However, E2, LH, and FSH were detectible in three Dax1-/Y mice and their levels were in a range that could be considered 'normal' (Fig. 3A,B). Examples of ovarian histology and steroidogenic gene-expression from a control female, an acyclic Dax1-/Y mouse, and a cyclic Dax 1-/Y mouse are provided in Fig. 3C. The acyclic Dax1-/Y mouse had underdeveloped ovaries with few mature follicles or corpora lutea (Fig. 3C). It also had dramatically reduced expression of key steroidogenic genes (Cyp19a1, Hsd3b1, 
A

C
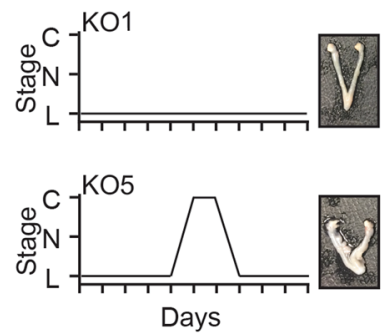

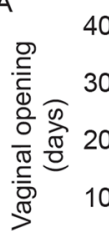

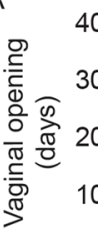

B

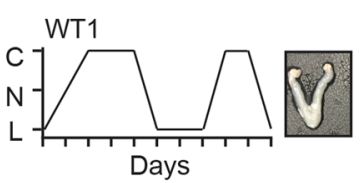

Figure 2. Intra-strain variability in reproductive phenotype of Dax1-/Y mice. (A) Age at first-day of puberty as measured by vaginal opening. WT $(\operatorname{Dax} 1+/+)$ females are presented as average $+/-$ SEM of four animals. (B) Representative estrous cycle and uterus/ovary morphology in a WT (Dax $1+/+)$ female mouse. (C) Individual estrous cycles and uterus/ovary morphology in each Dax1-/Y mouse. C, observation of cornified cells by vaginal cytology. $\mathrm{N}$ observation of nucleated cells by vaginal cytology. L observation of leukocytes by vaginal cytology.

A

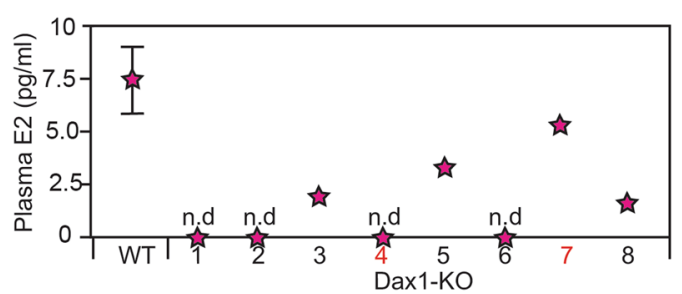

C
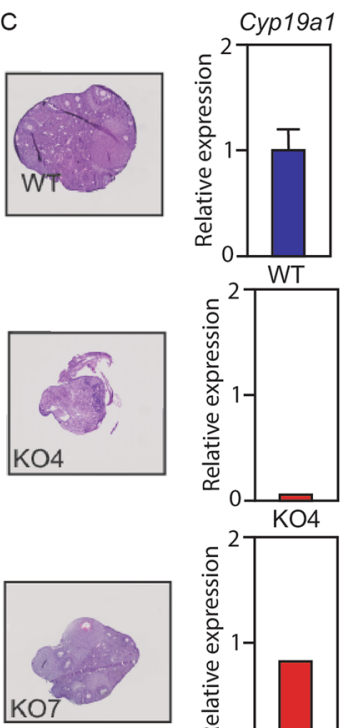
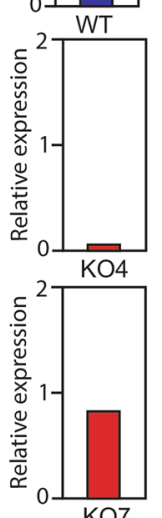

$\mathrm{KO}$

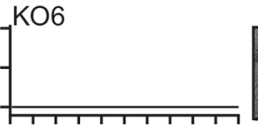

Days
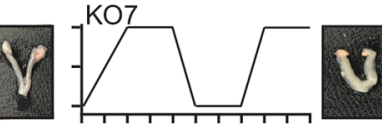

Days
B

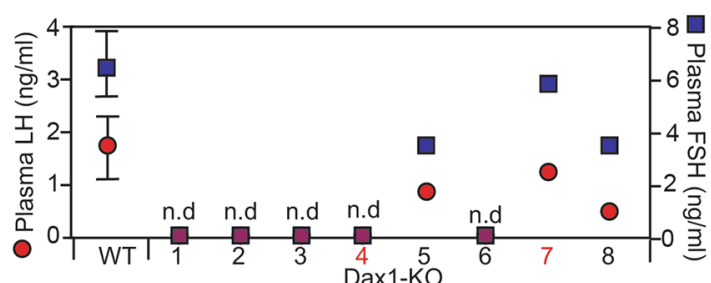

$\mathrm{D}$
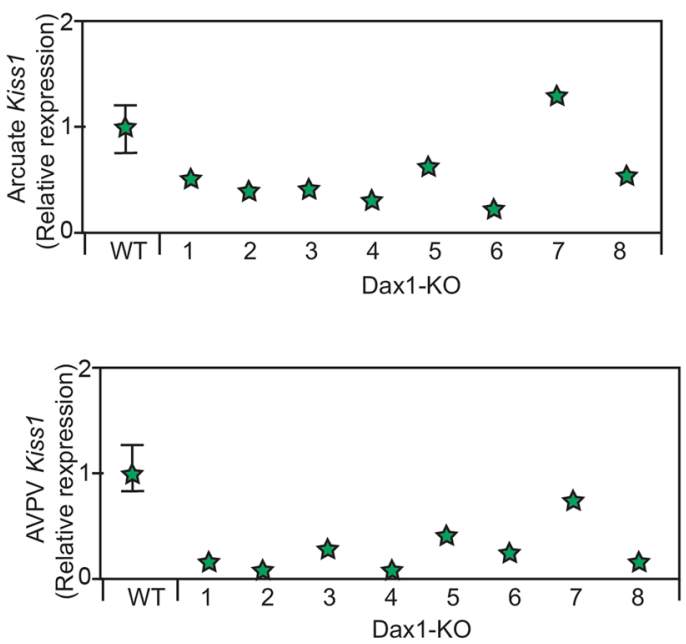

Figure 3. Dax $1-/ Y$ mice respond to ovarian estrogen production. (A) plasma estradiol levels. (B) Plasma LH and FSH levels in four control females, presented as average $+/-$ SEM, and eight individual Dax $1-/ Y$ mice. (C) Representative ovarian histology and ovarian steroidogenic gene expression in four WT $($ Dax $1+/+)$ female mice (gene expression presented as average +/-SEM), in an acyclic Dax 1-/Y mouse (KO4), and in a cyclic Dax1-/Y mouse (KO7). (D) Kiss1 gene-expression in the arcuate and AVPV hypothalamus in four control females, presented as average $+/-$ SEM, and eight individual Dax1-/Y mice. 
and Cyp17a1) (Fig. 3C). By contrast, the cyclic Dax1-/Y mouse had overtly normal ovaries and expression of Cyp19a1, Hsd3b1, and Cyp17a1 was comparable to controls (Fig. 3C).

Kisspeptin (Kiss 1) is a neuropeptide that controls fertility by signalling to gonadotropin-releasing hormone $(\mathrm{GnRH})$ neurons. In female mice, plasma gonadotropins (FSH and $\mathrm{LH}$ ) are controlled by kiss1-neurons in the arcuate nucleus (Arc) and anteroventral periventricular nucleus (AVPV) of the hypothalamus respectively. Hypothalamic Kiss1 expression was broadly in concordance with plasma gonadotropins in Dax 1-/Y mice. While the majority of these animals had lower Kiss1 expression than control mice, some had broadly normal Kiss1 gene expression in both the Arc and AVPV (Fig. 3D). Perturbations in Kiss1-expression in Dax1-/Y mice were more dramatic in AVPV than in the Arc (Fig. 3D). Furthermore, there is apparent concordance between plasma E2 levels, plasma gonadotropins, and hypothalamic Kiss 1-expression in individual Dax1-/Y mice (Fig. 3A,B,D). This suggests that the hypothalamus of $\operatorname{Dax} 1-/ Y$ mice is able to respond normally to ovarian sex steroids.

Taken together, these data provide the first evidence of dramatic intra-strain variation in markers of fertility in sex-reversed chromosomal-XY mice lacking the nuclear receptor Dax1. They also provide additional evidence that the primary defect in these animals is ovarian. Finally, we show that a minority of these sex-reversed chromosomal-XY mice can undergo puberty, maintain pregnancy, and give birth to live offspring.

\section{Discussion}

Our results confirm that the C57BL/6J background is highly sensitive to male-to-female sex-reversal ${ }^{10}$. Dax $1-/ Y$ mice on this strain present at weaning as phenotypic females. However, there is marked inter-individual variation in reproductive phenotypes. Most Dax $1-/ Y$ mice are infertile but a minority can give birth to live offspring.

It has been suggested that differences in reproductive phenotype amongst different strains of Dax $1-/ Y$ mice is due to the relative 'strength' (amount of transcript) of the male-determining Sry allele ${ }^{6,7}$. Indeed, overexpression of Sry in a strain susceptible to sex-reversal prevents ovary formation in Dax $1-/ Y$ mice $^{7}$. However, Sry mRNA expression has been shown to be variable even within-strain during the early events of testicular development ${ }^{7}$, and therefore provides a logical mechanistic explanation for the intra-strain variation in phenotypes observed here. Unfortunately, it is not currently possible to correlate early developmental expression of Sry in the testes to the degree of sex-reversal observed in adult Dax1-/Y mice. In addition, variability in sex-reversal has been observed on a number of other occasions in other models and possibly involves stochastic expression of pro-ovary genes ${ }^{11,12}$.

We found that most Dax1-/Y mice present with apparent hypothalamic hypogonadism. They have low plasma gonadotropins, and low estradiol levels. However, there was good concordance between plasma E2, hypothalamic Kiss1-expression, and circulating gonadotropins amongst individual Dax1-/Y mice (Fig. 3). This suggests that hypothalamic hypogonadism in these animals is secondary to ovarian dysfunction. In other words, individual Dax 1-/Y mice that are able to produce ovarian E2 are able to respond to it appropriately. Furthermore, the fact that Kiss1-expression in the AVPV is more dramatically affected than Kiss1-expression in the Arc of Dax 1-/Y mice is consistent with the hypothesis that the AVPV is a female-predominant nucleus which develops under the control of estrogen.

Although two out of a total of 18 Dax $1-/ Y$ mice gave birth, they each produced only one offspring, and neither animal survived beyond post-natal day two. As such, although a minority of Dax $1-/ Y$ mice have markers of fertility that are broadly in line with control females, they are still dramatically sub-fertile. The reasons for this are currently unclear but are probably due to a combination of factors that are likely a result of abnormal ovarian development. However, the mechanistic presence of a Y chromosome has also been suggested to inhibit meiosis in females ${ }^{13-15}$. Indeed, while we did detect DNA from the $\mathrm{Y}$ chromosome in an ovary from a sex reversed Dax1-/Y mouse that gave birth, this does not exclude the possibility that some ovarian cells in this animal may have lost the $\mathrm{Y}$ chromosome $(\mathrm{XO})$.

This work demonstrates that a minority of Dax $1-/ Y$ mice can produce live offspring without any assisted fertility treatment. It adds to the small number of reports of live-birth from sex-reversed XY mice (for example ${ }^{16-19}$ ). There are reports of fertility in mutant XY sex-reversed wood lemmings (Myopus shisticolor) ${ }^{20}$, and XY-female horses ${ }^{21}$. In addition, we are aware of one remarkable report of a fertile woman with normal ovaries and a predominantly XY karyotype ${ }^{22}$. As such, our observations in Dax1-/Y mice support the notion that XY sex-reversal is not formally incompatible with reproduction. However, our data also suggest that the inter-individual milieu of mechanical and development complications caused by the presence of the Y-chromosome makes successful reproduction highly unlikely.

\section{Methods}

Mice. All procedures were conducted in accordance with the U.K Animals (Scientific Procedures) Act 1986, with ethical approval from the Institutional Animal Care and Welfare Committee at Imperial College London. Mice were housed under standard conditions under a $12 \mathrm{~h}$ light-dark cycle and with free access to water and soyfree diet. C57BL/6J mice with deletion of $\operatorname{Dax} 1$ (NrOb2) were generated essentially as described previously and had undergone between 8 and 12 back-crosses on the C57BL/6J line ${ }^{7}$.

Mating experiments. Individual female Dax $1+/+$ mice, and Dax $1-/ Y$ mice were housed with a proven stud-male. Mating-success was determined by the presence of a vaginal plug in the morning during a ten-day mating experiment.

Puberty onset and estrus cyclicity. The day of vaginal opening (first day of puberty) and the stage of the estrous cycle were determined by cytology using standard methods.

Hormone analysis. Plasma estradiol was determined by ELISA (R\&D Systems). Plasma LH and FSH were determined by the University of Virginia Center for Research in Reproduction Ligand Assay and Analysis Core. 
Tissue collection and gene expression analysis. Tissues were collected in diestrus and either snap-frozen in liquid nitrogen or placed in buffered formalin for later histological analysis. Ovaries were dehydrated in ethanol and mounted in paraffin blocks. Sections were mounted on glass slides and stained using haematoxylin and eosin by standard methods. Ovarian and hypothalamic gene expression were determined following RNA extraction (Ambion PureLink), cDNA synthesis (Invitrogen), SYBR-Green qPCR (Sigma-Aldrich) and expressed as relative expression after correcting for internal control. Cyclophilin was used as an internal control. Primer-sequences for gene expression are available on request.

Statistical analysis. Statistical analysis were performed where appropriate by students t-test, with a $\mathrm{p}<0.05$ being considered statistically significant.

\section{Data availability}

All data will be made available upon reasonable request.

Received: 13 May 2019; Accepted: 2 January 2020;

Published online: 03 February 2020

\section{References}

1. Mangelsdorf, D. J. et al. The nuclear receptor superfamily: the second decade. Cell 83, 835-839 (1995).

2. Crawford, P. A., Dorn, C., Sadovsky, Y. \& Milbrandt, J. Nuclear receptor DAX-1 recruits nuclear receptor corepressor N-CoR to steroidogenic factor 1. Mol Cell Biol 18, 2949-2956 (1998).

3. Ludbrook, L. M. \& Harley, V. R. Sex determination: a 'window' of DAX1 activity. Trends Endocrinol Metab 15, 116-121, https://doi. org/10.1016/j.tem.2004.02.002 (2004).

4. Ikeda, Y. et al. Comparative localization of Dax-1 and Ad4BP/SF-1 during development of the hypothalamic-pituitary-gonadal axis suggests their closely related and distinct functions. Dev Dyn 220, 363-376, https://doi.org/10.1002/dvdy.1116 (2001).

5. Iyer, A. K. \& McCabe, E. R. Molecular mechanisms of DAX1 action. Mol Genet Metab 83, 60-73, https://doi.org/10.1016/j. ymgme.2004.07.018 (2004).

6. Meeks, J. J., Weiss, J. \& Jameson, J. L. Dax1 is required for testis determination. Nat Genet 34, 32-33, https://doi.org/10.1038/ng1141 (2003).

7. Bouma, G. J. et al. Gonadal sex reversal in mutant Dax1 XY mice: a failure to upregulate Sox9 in pre-Sertoli cells. Development 132, 3045-3054, https://doi.org/10.1242/dev.01890 (2005).

8. Yu, R. N., Ito, M., Saunders, T. L., Camper, S. A. \& Jameson, J. L. Role of Ahch in gonadal development and gametogenesis. Nat Genet 20, 353-357, https://doi.org/10.1038/3822 (1998).

9. Vazquez, M. J. et al. SIRT1 mediates obesity- and nutrient-dependent perturbation of pubertal timing by epigenetically controlling Kiss1 expression. Nat Commun 9, 4194, https://doi.org/10.1038/s41467-018-06459-9 (2018).

10. Munger, S. C., Natarajan, A., Looger, L. L., Ohler, U. \& Capel, B. Fine time course expression analysis identifies cascades of activation and repression and maps a putative regulator of mammalian sex determination. PLoS Genet 9, e1003630, https://doi.org/10.1371/ journal.pgen.1003630 (2013).

11. Harris, A. et al. ZNRF3 functions in mammalian sex determination by inhibiting canonical WNT signaling. Proc Natl Acad Sci USA 115, 5474-5479, https://doi.org/10.1073/pnas.1801223115 (2018).

12. Larney, C., Bailey, T. L. \& Koopman, P. Switching on sex: transcriptional regulation of the testis-determining gene Sry. Development 141, 2195-2205, https://doi.org/10.1242/dev.107052 (2014).

13. Obata, Y., Villemure, M., Kono, T. \& Taketo, T. Transmission of Y chromosomes from XY female mice was made possible by the replacement of cytoplasm during oocyte maturation. Proc Natl Acad Sci USA 105, 13918-13923, https://doi.org/10.1073/ pnas.0802680105 (2008).

14. Taketo, $\mathrm{T}$. The role of sex chromosomes in mammalian germ cell differentiation: can the germ cells carrying $\mathrm{X}$ and $\mathrm{Y}$ chromosomes differentiate into fertile oocytes? Asian J Androl 17, 360-366, https://doi.org/10.4103/1008-682X.143306 (2015).

15. Vernet, N. et al. The expression of Y-linked Zfy2 in XY mouse oocytes leads to frequent meiosis 2 defects, a high incidence of subsequent early cleavage stage arrest and infertility. Development 141, 855-866, https://doi.org/10.1242/dev.091165 (2014).

16. Eicher, E. M., Washburn, L. L., Whitney, J. B. 3rd \& Morrow, K. E. Mus poschiavinus Y chromosome in the C57BL/6J murine genome causes sex reversal. Science 217, 535-537 (1982).

17. Lovell-Badge, R. \& Robertson, E. X. Y. female mice resulting from a heritable mutation in the primary testis-determining gene, Tdy. Development 109, 635-646 (1990).

18. Kuno, J. et al. Generation of fertile and fecund F0 XY female mice from XY ES cells. Transgenic Res 24, 19-29, https://doi. org/10.1007/s11248-014-9815-y (2015).

19. Kuroki, S. et al. Epigenetic regulation of mouse sex determination by the histone demethylase Jmjd1a. Science 341, 1106-1109, https://doi.org/10.1126/science.1239864 (2013).

20. Liu, W. S., Eriksson, L. \& Fredga, K. XY sex reversal in the wood lemming is associated with deletion of Xp21-23 as revealed by chromosome microdissection and fluorescence in situ hybridization. Chromosome Res 6, 379-383 (1998).

21. Sharp, A. J., Wachtel, S. S. \& Benirschke, K. H-Y antigen in a fertile XY female horse. J Reprod Fertil 58, 157-160 (1980).

22. Dumic, M. et al. Report of fertility in woman with predominantly 46,XY karyotype in family with multiple disorders of sexual development: review of prismatic case. Mt Sinai J Med 75, 168-169, https://doi.org/10.1002/msj.20046 (2008).

\section{Acknowledgements}

W.S.D. is funded by an NIHR Research Professorship. B.M.O. is supported by a Sir Henry Dale Fellowship jointly funded by The Wellcome Trust and The Royal Society (105545/Z/14/Z). This article presents independent research supported by the NIHR at Imperial College Healthcare NHS Trust. The Section of Endocrinology and Investigative Medicine is funded by grants from the MRC, BBSRC, NHIR, and Integrative Mammalian Biology (IMB) Capacity Building Award, and FP7- Health- 2009-241592 EuroCHIP grant and is supported by the NIHR Biomedical Research Centre Funding Scheme. The views expressed are those of the authors and not necessarily those of the funders, the NHS, the NIHR, or the Department of Health.

\section{Author contributions}

I.F.F., A.M., and B.M.O. performed research. W.S.D., K.G.M. and B.M.O. supervised research and wrote the paper. 


\section{Competing interests}

The authors declare no competing interests.

\section{Additional information}

Correspondence and requests for materials should be addressed to W.S.D. or B.M.O.

Reprints and permissions information is available at www.nature.com/reprints.

Publisher's note Springer Nature remains neutral with regard to jurisdictional claims in published maps and institutional affiliations.

(c) (i) Open Access This article is licensed under a Creative Commons Attribution 4.0 International License, which permits use, sharing, adaptation, distribution and reproduction in any medium or format, as long as you give appropriate credit to the original author(s) and the source, provide a link to the Creative Commons license, and indicate if changes were made. The images or other third party material in this article are included in the article's Creative Commons license, unless indicated otherwise in a credit line to the material. If material is not included in the article's Creative Commons license and your intended use is not permitted by statutory regulation or exceeds the permitted use, you will need to obtain permission directly from the copyright holder. To view a copy of this license, visit http://creativecommons.org/licenses/by/4.0/.

(c) The Author(s) 2020 